\title{
CONRAD D'URACH, \\ DE L'ORDRE DE CITEAUX, LÉGAT EN FRANCE ET EN ALLEMAGNE.
}

\begin{abstract}
DARMI les familles illustres de l'Allemagne du moyen âge, celle des seigneurs d'Urach ou d'Achalm occupe un des premiers rangs. Elle doit son origine à Egon ou Eginon $\mathrm{I}^{\mathrm{er}}$, qui vivait au XI' siècle; un de ses fils, Gebhard, devint abbé de Hirschau, puis évêque de Spire. L'aîné des fils d'Eginon Ier s'appela du même nom que son père, et ce nom fut dès lors celui de tous les chefs de la famille. Un frère d'Egon III, appelé aussi Gebhard, occupa, de I I 3 I à I I4 I, le siège épiscopal de Strasbourg. Une de ses sœurs, Alberade, devint abbesse à Lindau, une autre, Wilhide, épousa le comte Frédéric de Zollern, dont la race était appelée à une haute illustration. Egon III eut pour fils Egon IV, surnommé le Barbu (r), qui mourut en 1230. Il avait épousé une personne d'une naissance aussi illustre que la sienne, Agnès, de la famille de Zähringen. Comme les Urach, les Zähringen avaient donné plusieurs membres de leur famille à l'Église, entre autres Rodolphe ou Raoul, qui devint évêque de Liége en I 68 et mourut en I I9I, dans le Brisgau, son pays d'origine, au retour de la croisade. Agnès de Zähringen avait un frère, Berthold V, qui mourut en février I 2 I8, sans postérité. En II97, à la mort de l'empereur Henri VI, Berthold, un des plus puissants seigneurs de son temps, fut élu empereur d'Allemagne par quelques seigneurs, sous l'influence de l'archevêque de Cologne. Mais il dut abandonner ses prétentions; il n'en demeura pas moins, jusqu'à sa mort, un des personnages les plus considérables de la Germanie.

Du mariage d'Egon le Barbu avec Agnès de Zähringen naquirent plusieurs enfants. L'aîné, Egon, devint dans la suite comte et seigneur de Fribourg.Le troisième, appelé Berthold, comme son oncle,
\end{abstract}

I. Mit dem Bart, en allemand. ou der Bärtige (Stälin, Wurtembergische Geschichte, II, 457. Il existe un document de l'an 1228 , avec le sceau du quatrième comte d'Urach. Il y est représenté portant une longue barbe. (Stälin, op. cit.) 
revêtit l'habit religieux, et devint successivement abbé de Tennenbach, de Lützel et de Salem. Le quatrième portait le nom de Rodolphe, comme son grand oncle, l'évêque de Liége. Déjà avancé en âge, il se retira dans un monastère, celui de Bebenhausen, où il finit ses jours. La famille comptait encore parmi ses membres un autre fils, appelé aussi Berthold et deux filles, Hildwide, qui épousa le comte de Pfirdt, et Yolande, qui devint la femme du comte de Neûchatel (I).

Nous avons jusqu'ici passé sous silence le nom du plus illustre des enfants de cette famille, Conrad. Jeune encore, il devint doyen du chapitre de Saint-Lambert de Liége, un des plus célèbres de cette époque; la plus haute noblesse seule en ouvrait l'accès. Mais quelques années plus tard, il dit adieu au monde et se retira à l'abbaye cistercienne de Villers, une des plus importantes fondations de saint Bernard en Belgique. Il y devint prieur, puis abbé, jusqu'au moment où il fut appelé au gouvernement de l'abbaye de Clairvaux. Son séjour n'y fut pas de longue durée : 2 ans $1 / 2$ après, il devint supérieur général de tout l'ordre de Cìteaux. De plus grands honneurs l'attendaient encore : il serait devenu archevêque de Besançon si Honorius III, élu pape depuis peu, ne l'eût élevé au cardinalat. Il fut alors chargé de deux légations importantes, l'une en France, pour y lutter contre l'hérésie albigeoise, l'autre, en Allemagne, pour y prêcher la croisade. A son retour à Rome, les cardinaux lui offrirent la tiare pontificale; mais il la refusa. Six mois plus tard, sur le chemin de la Terre-Sainte, la maladie l'emporta en quelques jours.

Il est difficile de fixer la date exacte de la naissance de Conrad. Il apparait pour la première fois dans l'histoire en I 195 , comme doyen du chapitre cathédral de Liége (2). Dans ce cas, il serait

I. Stälin, Wurtembergische Geschichle, II, 452 et $28 \mathrm{r}$.

2. Buldstin de la commission royale d'histoire de Belgique, Série IV, 1, P. I24. Le P. Gloning. (Conrad von Urach, Cardinalbischof von Porlo und snncta Rufina, Augsburg, 190I) dit que Conrad devint doyen en I 193, en se basant sur le Gallia Christiana, III, coll. 927. Le Gallia s'autorise d'une charte donnée par l'empereur et adressée à * dilecto et fideli suo Conrado decano B. Lamberti in Leodio \%. Cette charte est donnée en entier dans Bormans et Schoolmesters (Cartulaire de "'Eglise St-Lambert à Liége, mais les auteurs ont avec Stumpf (Dit Reichskanzler, III, 281) fixé la date de ce document au mois de mai i 96 . MM. Bormans et Schoolmeesters (loc. cit.) ainsi que la Commission Royale d'histoire disent que Conrad devint doyen en I 195 . Ils sont d'accord avec la liste publiée dans les Analectes de lhistoire ecclesiastique de Belgique, t. XXV, où nous ne voyons pas le nom de Conrad figurer avant cette année. Conrad est, par erreur, désigné par eux sous le nom de Conrad de Fürstemberg. 
difficile d'admettre, avec le P. Gloning, que Conrad vit le jour en I 79 ou I 80 : il n'aurait eu alors que is ans. De plus, lorsqu'en I 200, quelques chanoines voulurent faire de lui le successeur de $\mathrm{S}$. Lambert, il aurait atteint à peine sa vingtième année. Il faut aussi ajouter que Berthold, plus jeune que Conrad, devint abbé de Tennenbach en I 206. Tout ce que nous savons de son adolescence, c'est qu'il fut envoyé à Liége, fameuse par ses écoles, où il trouva un puissant protecteur en son grand-oncle, l'évêque Raoul de Zähringen ( $\mathrm{I}$ ). Mais à la fin du XIIe siècle, des événements imprévus donnèrent à son avenir une direction différente.

En I I97, l'empereur Henri VI descendit dans la tombe, ne laissant qu'un fils âgé de trois ans, le futur empereur Frédéric II. Philippe, duc de Souabe, oncle et tuteur du jeune prince, se trouvait alors en Italie. Il n'eut rien de plus empressé que de repasser en Allemagne, pour y soutenir les intérêts de sa maison. La plupart des seigneurs du Nord et de l'Est de l'Allemagne se rangèrent à ses côtés, mais les princes des bords du Rhin étaient loin de partager ses vues. Parmi ceux-ci, l'archevêque de Cologne, Adolphe, dont la puissance égalait l'habileté, se déclara ouvertement contre les parents de l'empereur défunt et gagna ses voisins à la cause de son candidat, Berthold de Zähringen, dont le récent conflit avec Conrad de Souabe, un autre frère de Henri, était encore présent à la mémoire de tous. Berthold avait le second rang parmi les princes, le souverain de Bohême seul passait avant lui. Sa fortune était immense, et ses grands talents d'administrateur l'avaient rendu célèbre. On lui proposa la couronne, et dès d'abord, il l'accepta. Quant à Philippe de Souabe, qui travaillait toujours pour son jeune neveu, il ne répondit pas aux avances du parti qui voulait l'élever au trône impérial. L'archevêque de Cologne et ses amis fixèrent un jour pour l'élection de Berthold, qui devait venir en armes à Andernach. Il faut croire cependant qu'il inspirait peu de confiance à ses partisans, car il dut leur livrer comme otages ses deux neveux Conrad et Berthold, qui étudiait peut-être aussi à Liége. Mais ensuite, il se ravisa. L'Allemagne du Nord et de l'Est lui étant opposée, son élection amènerait certainement la guerre, et la guerre lui coûterait beaucoup d'argent. Or, il était avare, et, réflexion faite, il annonça à ses partisans qu'il abandonnait ses prétentions. Quant à ses neveux, il ne s'en occupa pas davantage. Se trouvant ainsi prisonniers, ils firent, d'après quelques-uns, le vœu d'entrer en reli-

1. Egidii Aurævallis Gesta Episcoporum Leodiensium, Pertz. SS. 25, 121. 
gion s'ils étaient délivrés; d'après d'autres, moins dignes de foi, ils furent contraints de se consacrer à l'état ecclésiastique. Quelque temps après, Berthold entrait à Lützel, Conrad frappait à la porte du monastère de Villers ( $\mathrm{s}$ ).

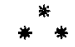

Peu de temps après son noviciat, Conrad fut élevé à la dignité de prieur, et en 1209, quand mourut l'abbé Charles de Seyne, les moines lui confièrent la direction de leur abbaye. Dans ces deux charges, il s'acquit l'estime de tous par sa discrétion, sa prudence et son zèle dans l'accomplissement de ses devoirs ( $\left.{ }^{2}\right)$. Ce fut sous lui qu'un cistercien, appelé aussi Conrad, et qui avait été évêque avant son en-

I. de Theux, dans son Hisloire du Chapitre de Saint-Lambert (1, 204) dit que, vers 1203 , Conrad se retira à l abbaye de Villers. On peut fixer la date d'une manière plus précise. Le Cartulaire de Saint-Lambert (1, 127, note) dit que, en I 200, Waltère de Ravenstein était déjà doyen, et Thimister (Cartulaire de Saint-Paul, pp. 29 et 30 ) donne deux chartes, l'une de 1200 , l'autre de 1202 où Walter figure comme doyen ( majoris ecclesiæ). D'après certains auteurs, tout à fait dignes de foi (Chronique de lillers, Pertz, SS. 25, 198 et Albricus Pertz 23, 878), Conrad était encore novice à Villers quand il fut question de l'élever à l'évêché de Liége. $\mathrm{Ce}$ ne peut être qu'en I200, lors de l'élection de Hugues de Pierrepont, qui mourut en 1229. On peut donc fixer à 1199 l'entrée du doyen de St-Lambert au monastère. S'il faut en croire certains récits, Conrad, étant novice, aurait été le héros de cette aventure. \& Un frocre convers d'Aulne,nommé Simon, possédant le don de prophétie, se trouvait avec le moine Waltère, qui succéda \&̀ Conrad comme abbé de Viliers, plusieurs xeligieux et frères convers de ce monastère, dans une église où ils assistèrent à la messe. Pendant le canon, Simon vit, debout devant lui, Conrad, qui était cependant loin de la, portant sur sa tête une couronne d'or. Il eut connaissance des pensées qui l'occupaient et des prières qu'il faisait alors à Villers. La messe finie, le frère Simon dit au moine Waltère. \& Quand vous verrez votre novice Conrad, dites-lui d'être sur ses gardes, car cette année, telle espèce de tentation le tourmentera. Pendant la messe, il a eu telles pensées et dit telles prières, il deviendra un personnage important dans votre ordre. Ensuite, quand Waltère revit Conrad, il lui demanda quelles pensées et quelles prières l'occupaient d'ordinaire pendant la messe. Conrad les lui indiqua et l'autre ajouta: Tel dimanche, pendant la messe, quelles furent vos pensées? - Pourquoi donc, repartit Conrad, m'interroger avec tant de soin sur ce sujet? - Répondez-moi d'abord, je vous indiquerai le motif ensuite." - Le novice lui exposa tout ce à quoi il avait pensé et Waltère fut fort étonné de voir que c'était en tout point ce que Simon lui avait raconté. II le dit à Conrad, lui parla de cette tentation et l'avertit de prendre garde, de peur d'être trompé par le démon. Malgré toutes ces précautions, Conrad ne put éviter d'être tenté comme Waltère le lui avait prédit s. Nous nous contenterons d'ajouter, par rapport à l'authenticité de ce fait les remarques suivantes: Il ne se trouve pas dans le manuscrit, I (Bruxellensis), le manuscrit 2 seul en parle. Le moine de Villers qui nous livre ce récit, «aime le merveilleux et exagère l'intervention du surnaturel. \$(Balau, Mémoires couronnés publiźs par l'Acadénie Royale de Belgique, tome LXI, p. 48I.) Ce qui nous est raconté par les Gesta Sanctorum Villariensium (Pertz, SS. 25, 223) est ígalement, el dans les mêmes termes dans Césaire d'Hesterbach, Dialogus Miraculorum, I, p. I 53, et Césaire paraît aimer les récits de ce genre. C'est peut-être lui qui en est l'unique source. Il dit de Conrad : ( $N u n c$ episcopus Portuensis. ) Ces détails lui auraient été donnés par le moine Waltère lui-même «a cujus ea ore audivi », p. 553 . Il suffit d'ailleurs d'une simple lecture pour s'apercevoir que cette histoire manque totalement de naturel.

2. Chron. Villar. ap. Pertz, SS. 25, p. 198. 
trée au monastère, bénit le cimetière de Villers ( 1 . Le nouvel abbé sut par ses grandes qualités, mériter l'estime des seigneurs voisins. Guillaume d'Opprebais et sa femme Élisabeth donnèrent à l'abbaye l'argent nécessaire pour acheter les cierges qui devaient servir au saint Sacrifice, afin d'avoir part aux bonnes œuvres du monastère (2). Conrad compta aussi parmi ses amis le comte de Namur, Philippe le Noble. Le chapitre de Notre-Dame de Huy avait des démêlés avec ce seigneur au sujet de la propriété du bois de Profondeville. Conrad prit part à l'arbitrage qui régla ce différend (3). Quand le comte fonda une prébende à l'église de Saint-Aubain, à Namur, en I 2 I2, Conrad souscrivit l'acte de fondation $\left({ }^{4}\right)$. Conrad fut aussi présent à la mort de Philippe. Celui-ci se trouvait à son château de Blaton, entre Ath et Condé, quand il sentit sa fin prochaine. Il manda les abbés de Cambron, de Villers, de Marchienne et de Valenciennes pour l'aider à bien mourir. Il voulait qu'on le traînât par les chemins, la corde au cou, pour expier ses fautes, mais les abbés firent opposition à un acte aussi peu raisonnable. Ce furent ces quatre abbés que le comte choisit pour ses exécuteurs testamentaires ( 1212 ) (5).

La même année, les abbés de Villers et du Val-Saint-Lambert, choisis pour juges, décidèrent que l'abbaye d'Aulne possédait légitimement une terre située à Awans et la dìme de cette terre, et interdisait à Lithold de Noville d'élever de nouvelles réclamations. Conrad fut aussi témoin de l'acte par lequel le duc Henri de Lothier donna une partie de la dime de Dion pour doter la même abbaye d'Aulne $\left({ }^{6}\right)$. Un différend avait surgi entre les religieuses cisterciennes du Val-Notre-Dame, près de Huy, et les prémontrés de Wanze, qui déniaient aux moniales le droit d'habiter encore leur monastère (7). Conrad fut assez heureux pour obtenir de l'abbé de Floreffe l'abandon de ces prétentions. Mais le temps était arrivé où Conrad devait être élevé à de plus hautes dignités.

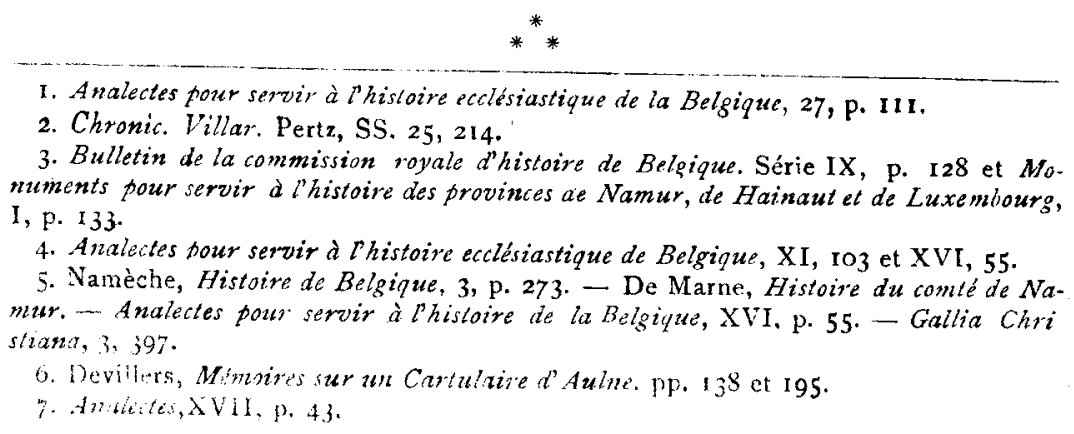


En I 2 I 4, vers la fin de l'année, l'abbé Guy de Clairvaux mourut. Quand on voulut lui donner un successeur, les suffrages se réunirent sur l'abbé de Villers ( $\mathrm{I}$ ). Clairvaux avait eu à sa tête, un siècle auparavant, l'illustre saint Bernard. Cette abbaye était, en même temps que Morimond, Pontigny et la Ferté, une des «filles》 de Cîteaux et son abbé, un des personnages les plus considérables de l'ordre. Un grand nombre d'abbayes étaient sous sa juridiction. Les années du gouvernement de Conrad furent heureuses: ce fut notamment sous lui que l'on vit entrer à Clairvaux un puissant seigneur, Guillaume de Rixelles, qui donna des biens considérables au monastère ( $\left.{ }^{2}\right)$. Conrad parut au chapitre général de I 2 I $4\left(^{(3)}\right.$. Ce chapitre régla un différend important entre les abbés de Dalon et de Bonneval, qui se disputaient la prééminence sur deux autres abbayes et donna à l'abbé de Cîteaux et aux quatre grands abbés le pouvoir de retirer les décrets des chapitres généraux, s'ils le jugeaient nécessaire. En I2 I5, cette décision fut renouvelée.

Sur ces entrefaites, le Pape Innocent III convoqua au Latran un concile acuménique (4). On vit se diriger vers Rome un grand nombre de prélats de toutes nations. L'abbé de Cîteaux s'y rendit, accompagné des abbés de Clairraux, de Pontigny, de la Ferté et de Morimond.

Ce concile de Latran devait avoir une grande importance sur le gouvernement des ordres religieux. Jusque-là, dans l'ordre cistercien seulement, avaient lieu, chaque année, des chapitres généraux où se réunissaient les abbés de tout l'ordre pour la réglementation des affaires importantes et la réforme des abus. Le concile étendit à tous les ordres religieux cette sage institution (5).

I. Le Gallia christiana dit que Conrad, étant abbé de Clairvaux, souscrivit au mois d'Avril à une bulle réglant un accord entre les abbayes de Montiéramey, au diocèse de Troyes et d'Arrivour, au même diocèse. Le P. Gloning (op. cit., p. 22) croit, avec raison, que cette bulle est de l'année suivante. Conrad ne fut certainement que deux ans et demi ì Clairvaux. (Manrique, Annales cisterciennes, IV, 47, et d'Arbois de Jubain. ville, Etudes sur létat intérieur des abbayes cisterciennes auX $X I^{\mathrm{e}}$ at $X I J^{\mathrm{e}}$ siècles, p. 354.) Or, il serait, dans l'hypothèse du Gallia, devenu abbé de Cîteaux, non pas en 1217 , comme tous les témoignages l'affirment, mais en 1216. Cfr. Chronicon Villariense, ap. Pertz, SS. pp. 197 et 198.

2. Gallia christiana, IV, 804 .

3. Gallia christiana, IV, 804 . - Martène et Durand, Thesaurus novus anecdotorum, IV, pp. 1313 et 1314 , où son nom figure parmi les signataires d'un décret.

4. La présence de Conrad à Rome nous est attestée par une lettre d'Honorius III. * Cum nuper in concilio generali, ad sedem apostolicam venientes, essetis in felicis re cordationis Dni Papze et predecessoris nostri presentia constituti (Manrique, IV. 85 , Cfr. Pressutti, Regesta Honorii III et Potthast, Regesta Fontif. Rom., p. 468, nc 5322.) 5. Les décrets des chapitres généraux cisterciens se trouvent dans Martène et Durand. Thesaurus novus anecdolorum, tome IV. Pour l'obligation imposée aux ordres religieux de tenir des chapitres, voir Corpus juris, ed. Richter, I. II, t. XXXV. De stalu monachorum, c. 7 . 
Mais le principal motif de la venue à Rome des abbés cisterciens était, semble-t-il, de terminer un différend qui existait entre eux.La constitution de l'ordre de Cîteaux était réglée, avant tout, par la fameuse Charte de Charité. Le chapitre troisième s'occupait des dissensions entre les abbés et des peines qui pourraient les frapper, s'ils manquaient à leurs devoirs. En cas de doute, la sentence était rendue par l'abbé de Cîteaux ou par ceux dont le jugement paraissait le plus équitable. L'ordre avait alors à sa tête un homme d'une grande vertu, mais peut-être trop autoritaire. Il trouvait que la Charte de charité lui donnait le pouvoir de reprendre les quatre grands abbés comme les autres, de les déposer même, si leurs fautes l'exigeaient. Les abbés lui reconnaissaient d'autant moins ce droit que la même charte leur confiait la visite canonique du monastère de Cîteaux. Ils répondaient que le consentement des trois autres était requis pour que le quatrième fût déposé. La coutume et la raison parlaient en leur faveur, car les prétentions d'Arnaud tendaient à faire disparaître toute différence entre les quatre grands abbés et les autres. D'autre part, en cas de déposition d'un abbé, la sentence paraîtrait certainement plus juste et plus raisonnable si l'abbé de Cíteaux et les trois autres agissaient de concert. Innocent III ne soumit pas la solution du différend aux pères du concile, pour ne pas le révéler; il préféra la soumettre à la sagesse et à la prudence de l'évêque d'Ostie. Sa mission était surtout d'amener entre les parties un accord librement consenti ( $\mathrm{I}$ ).

Le cardinal ne trancha pas la question et dut se borner à cette déclaration. Si l'abbé d'une des 4 grandes abbayes vient à mourir, l'abbé de Citeaux ira présider l'élection de son successeur. S'il veut déposer l'un des quatre abbés principaux, pour raison d'inconduite, il se rendra dans son abbaye, avec les abbés qu'il désignera. L'abbé accusé, prévenu quatorze jours d'avance, désignera d'autres abbés, à son choix ; l'abbé général prendra conseil des deux parties et décidera comme il le jugera bon. Toutes les difficultés n'étaient pas aplanies, mais Innocent III confirma au concile ce que le cardinal avait décidé. Il ajouta cependant : « l'abbé de Cîteaux exposera ses motifs devant tous les abbés assemblés et avec l'assentiment de tous ou de ceux dont le jugement lui paraitra le plus sain, si les avis se partagent, il donne sa décision. C'est lui qui décidera quelle opinion lui paraitra préférable. Mais si le contraire arrive, on en référera au chapitre général de l'ordre, qui aura le pouvoir d'amender. » Le

†. Voir Manrique, IV, 60. 
pape ne condamnait ainsi ni l'abbé Arnaud, ni ses adversaires ; personne n'était ni vainqueur, ni vaincu. Il arriva ce qu'il arrive de la plupart des demi-mesures : personne ne fut content. Mais comme l'ordre cistercien avait souffert de ces dissentiments, Honorius III, le successeur d'Innocent, crut devoir écrire aux cinq abbés \& de ne rien proposer ou décider, au chapitre général, qui pût être pour l'ordre un sujet de scandale ( ${ }^{r}$ ). » Les abbés s'abstinrent, en effet, d'agiter la question de leurs droits. L'abbé de Clairvaux continuait à mériter la confiance de son ordre. Étienne, comte de Bourgogne, ayant formé le dessein de fonder un monastère de cisterciens, Conrad fut désigné pour traiter cette affaire avec lui. Vers cette époque, il soumit le monastère de Baudeloo, en Belgique, à l'abbaye de Cambron, et régla un différend entre son ancienne abbaye de Villers et les prémontrés de Floreffe, qui reprochaient aux cisterciens d'avoir bâti des fermes à Villeret et Wierde $\left({ }^{2}\right)$, au mépris de leurs droits.

Le chapitre général, qui se tint sur la fin de l'année, était à peine terminé que les abbés de Cîteaux et de Clairvaux furent chargés par le pape d'une mission fort importante. Depuis longtemps déjà, la guerre durait entre la France et l'Angleterre. La mort de Jean-sansTerre, survenue en I2 I5, n'avait pas suspendu les hostilités; au contraire, Philippe-Auguste se réjouissait sans doute de voir le sceptre d'Angleterre aux mains d'un enfant. Innocent III avait, à plusieurs reprises, cherché à amener la paix, mais sans pouvoir y réussir. Honorius voulut tenter d'obtenir ce qui avait été refusé à son prédécesseur. Par une lettre datée du 6 décembre I 2 I 6 (3), le pape chargea les abbés Arnaud et Conrad de terminer le différend, si c'était possible. Cette mission était hérissée de difficultés. PhilippeAuguste était alors victorieux, et son fils Louis, qui devait lui succéder, était en Angleterre, maintenant ses prétentions contre les seigneurs anglais. De plus, « si le pape croyait ne pas pouvoir abandomner la cause des fils de Jean-sans-Terre, il ne voulait pas non plus s'aliéner un roi aussi puissant que Philippe-Auguste $\$\left({ }^{4}\right)$. Le pape enjoignait aux deux abbés de se rendre d'abord auprès du

1. Pressutti, op. cit., r, p. 3. - Manrique, IV, 85. - Potthast, Reg. Pont. Rom., p. $468, \mathrm{n}^{\circ} 5322$.

2. Statuta capituli generalis ord. cisterciensis. Martène et Durand, IV, col. 1317 et 1318 . - J. J. de Smet, Carlulaire de l'abbaye de Cimbron, i, I19. Histoire de l'abbaye de Flo. reffe, par le chanoine Barbier, II, p. 7I. Le chanoine Barbier dit que cette charte fut donnée vers I218. Il faut la reporter à une date antérieure; Conrad y est désigné sous le titre d'Abbas Clarevallensis et il cessa de l'être en I2r7.

3. Pressutti, Reg. Hon. III, I, 29. - Manrique, IV, 86. - Bouquet, Recueil des anciens historiens des Gaules, XIX, donne la lettre d'Honorius III en entier.

4. Manrique, $1 X, 86$. 
roi de France, pour lui demander de rappeler son fils, et de partir ensuite en Angleterre, pour prier ce dernier de consentir à la paix. Il ne semble pas que le succès ait couronné leurs efforts. $\mathrm{Au}$ commencement de l'année suivante, le pape se voyait obligé d'accorder à son légat en Angleterre les pouvoirs les plus étendus pour défendre le jeune roi contre le fils de Philippe-Auguste. De plus, il revint à la charge auprès du roi de France pour lui demander de renoncer à ses prétentions. Toutes les instances furent inutiles. Louis, continuant la guerre, se dirigea vers Lincoln. Il tenta de faire le siège de cette ville, mais les seigneurs anglais vinrent l'y rejoindre et lui liver bataille. Il fut vaincu et fait prisonnier. Au mois de juillet suivant, les Français étaient encore battus sur mer. On convint enfin d'une paix dont la durée ne fut pas longue. Le fils du roi de France fut mis en liberté moyennant une forte rançon ( $\urcorner$ ).

Arnaud et Conrad eurent la douleur d'être témoins, en Angleterre, de la sévérité que déployait le légat Galon à l'égard des monastères cisterciens. Il avait mis plusieurs couvents sous l'interdit, frappé de la suspense plusieurs abbés et leurs moines, en avait excommunié d'autres. Les deux abbés virent dans une pareille conduite une violation formelle des privilèges de leur ordre. Ils en appelèrent au pape, mais ce fut en vain. Le pape augmenta même les pouvoirs du légat pour qu'il pût continuer la guerre contre les Français ( ${ }^{2}$ ).

Après son voyage en Angleterre, Conrad était rentré à Clairvaux où il continua à gouvemer paisiblement son monastère. Ce fut vers cette époque que se fondèrent, sous son autorité, plusieurs abbayes Cisterciennes, notamment celles de Fontenelle, près de Valenciennes, d'Épinlieu (Locus Spinosus) et d'Essen ( ${ }^{3}$ ).

Les dissentiments entre les abbés duraient toujours. Au mois de mars 12 I 7 , le pape se vit obligé de confirmer l'accord imposé aux deux parties et approuvé au concile de 12 I 5 (4). Honorius ne condamnait ni l'abbé de Cîteaux ni les autres, et la question restait toujours pendante ; seul, l'abbé de la Ferté semblait renoncer à ses prétentions. Le pape rappelait aux abbés le mal que cette discorde pouvait produire dans l'ordre et les nombreux efforts tentés pour y porter remède. Il ajoutait qu'il maintenait dans toute sa rigueur, la constitution donnée par Innocent III et ordonnait à tous de l'observer. Ce fut pour ce motif peut-être qu'Arnaud, qui était déjà avancé en

I. Pour les lettres du pape au légat et à Philippe-Auguste, voir Bouquet, XIX, pp. 623 . $24,29,30,31$. Pour les événements de la guerre. Id. XIX, pp. 26I el sqq.

2. Bouquet, XIX, p. 616.

3. Manrique IV, 96 et 97.

4. Pressulti, Reg. Hon III, x, 75. - Manrique IV, 100. Polthast, p. 483, n. 5467. 
âge, résigna sa charge. Au commencement d'aviril 12 I 7 , les électeurs mirent à la tète de leur ordre l'abbé de Clairvaux. Conrad quitta bien à regret, ce semble, l'abbaye qu'il avait dirigée pendant deux ans et demi. Toute sa vie, il conserva pour elle un souvenir particulièrement affectueux. Il voulut que ses cendres y reposassent à côté de celles de saint Bernard ( $\mathrm{I}$ ). Plus tard, devenu cardinal, il donna à l'église de Clairvaux la tête du saint martyr Vincent $\left(^{2}\right)$ et y fit aussi transporter les corps des saints Eutrope, Zosime et Bonose, qui se trouvaient à Porto. Des objets précieux furent aussi légués par lui à son ancien monastère (3).

L'abbé de Cîteaux était déjà à cette époque l'un des personnages les plus importants de la chrétienté. Près de 600 monastères d'hommes se trouvaient sous sa juridiction. Les cisterciens possédaient des abbayes en Syrie, en Sicile, en Portugal et en Norvège (4). Conrad était en fonctions depuis peu quand il dut, en sa qualité d'abbé de Cîteaux, présider le chapitre général de l'ordre. Le pape Honorius avait accordé, cette année, à tous les abbés cisterciens réunis en chapitre, la faculté de dispenser leurs religieux qui étaient sous le coup d'une irrégularité $\left(^{5}\right)$. Ce chapitre général ne paraît pas avoir eu grande importance: les décrets qui y furent rendus se bornèrent à formuler certaines prescriptions pour que la règle fût observée et pour prévenir quelques abus ( $\left.{ }^{6}\right)$. Presque aussitôt après, l'abbé de Cìteaux se rendit à Toulouse. Il devait y rejoindre le comte Simon de Montfort, le vaillant défenseur des catholiques dans la guerre des Albigeois. Le concile de Latran avait lancé l'excommunication contre. Raymond de Toulouse, le chef des hérétiques, et avait confisqué ses terres au profit de Simon ( 7 ). Raymond s'était peu soucié de se soumettre, et la guerre continuait toujours. Le chapitre général de I 2 I 6 avait défendu sévèrement à tout cister-

\footnotetext{
1. Voir Migne, Pat. Lat., n 185 , c. 1766 .

2. Gallia Christiana, IX, p. 884; Acta Sanctorum, 22 janvier, 3, p. I2.

3. Manrique, IV, 327, cf. Migne, Pat. Lat., n' 1305 pp. 1768-69.

4. Gloning, op, cit., p. 27. Voir aussi dans Martène et Durand, les dispenses accordées aux abbés de Norvège, de Syrie et de Sicile de se rendre au chapitre, a cause de la longueur de la route, IV, 1320.

5. Pressutti, Reg. Hon. III.

6. Ces décrets se trouvent dans Martène et Durand, IV, I3I9, 20, 2 I.

7. Potthast, p. 439, no 5009. Cf. Vaissettc, Histoire du Languedoc. Bouquet, 19,598, et Migne, CEuvres d'Innocent $I I I$, vol. III, p, 992. Le pape écrivit aussi a Simon de Montfort pour lui signifier cette décision et le prier de la faire exécuter.
} 
cien de nuire, en aucune façon, aux intérêts du comte de Montfort. A la fin de l'année, Conrad se rendit auprès de lui. Le 3 novembre, il signa une charte datée de Toulouse (I). Il voulait sans doute porter aide au chef catholique qui assiégeait alors cette ville. Le chapitre décida l'année suivante, que les abbés de Bithaine, de Nerlac et de Chalivois se rendraient dans le territoire des Albigeois pour punir sévèrement les moines et les frères qu'ils trouveraient coupables. Trois d'entre eux, qui avaient aidé le comte de Foix et les Toulousains, reçurent ordre de venir à Cîteaux, recevoir le châtiment qu'il plairait à l'abbé général de leur infliger.

Le I $^{\mathrm{r}}$ décret du chapitre de I 2 I 8 décida, sur la proposition de Conrad, la récitation d'une prière qui, depuis, fut toujours en grand honneur parmi les cisterciens: le Salve Regina. Un autre défendait à tout moine d'accepter l'épiscopat sans le consentement de son abbé et de celui de Cìteaux ( 2 ). Le monastère de Trebnitz, en Pologne, que devait illustrer Ste Hedwige, fut incorporé à l'ordre ( ${ }^{\mathfrak{B}}$ ). Honorius III enjoignit aussi de célébrer solennellement, dans toutes les églises cisterciennes, la fête des saints Jean et Paul, et de mentionner leur nom dans les litanies des saints (4). Le pape écrivit encore plusieurs fois à l'abbé de Cìteaux. Révolté de la cruauté des Avignonnais, qui avaient tué et coupé en morceaux Hugues, prince de Baux, un des chefs catholiques, il invita personnellement Comrad à aider de tout son pouvoir Amaury, fils de Simon de Montfort. Philippe-Auguste, libre du côté de l'Angleterre, consentait maintenant à porter secours aux catholiques du Sud de la France. Honorius recommanda à l'abbé de Cîteaux, et aux évêques de Meaux et de Noyon de frapper des censures ecclésiastiques les adversaires du roi et de rassembler les vingtièmes dont une moitié devait être employée contre les Musulmans, l'autre contre les Albigeois. Manrique croit $\left({ }^{5}\right)$ avec beaucoup de vraisemblance, que le chapitre général de 2 I 8 avait décidé le départ de Conrad pour Rome où il devait se plaindre de certaines vexations infligées à l'ordre de Cîteaux. Vers la fin de cette année, l'abbé se mit en

1. d'Arbois de Jubainville, Etudes suy That interieur des abbayes cisterciennes aux $X I I^{e}$ et $X I I I^{\prime}$ sizcles, p. 138 .

2. Martène et Durand, IV, col. 1321-23. Ce chapitre défendit strictement aux abbés de célébrer la messe sur un autel couvert de tapis. Si cette prescription était violée, les religieux qui avaient apporté les tapis el l'abbé qui avait célébré la messe avaient à faire trois jours de pénitence, dont l'un, au pain et à l'eau.

3. Grünhagen, Schlesische Regesten, 1, IOI. - Franz Winter, Die Cistercienser des nordöstlichen Deutschlands.

4. Pressutti, I, p. 24I, Manrique 4,425; Potthast, p. 513, $\mathrm{n}^{\circ} 534^{2}$.

5. Manrique, IV, 150 . 
route pour la capitale du monde chrétien. C'est peut-être à son crédit auprès d'Honorius que son ordre dut l'obtention d'importants privilèges. Deux avaient rapport aux dìmes dont quelques-unes avaient, ce semble, été levées au mépris des ordonnances ecclésiastiques. Le troisième défendait aux légats ou à leurs procurateurs d'exiger de l'argent des monastères cisterciens, le quatrième leur interdisait d'excommunier, de suspendre ou de frapper d'interdit les religieux de Cîteaux sans une autorisation spéciale du pape. Des prêtres séculiers prétendaient que lorsqu'une personne soumise à leur juridiction spirituelle entrait en religion, elle devait leur payer le a mortuarium $\gg$ ou les frais de funérailles: le pape cassa cette prétention ( $\mathrm{r}$ ).

A Rome, la pourpre cardinalice attendait le général des cisterciens. Il avait été bien peu de temps, moins de deux ans, à la tête de son ordre. Les dissentiments qui s'étaient élevés entre le chef et les quatre abbés principaux ne s'étaient pas renouvelés, sans doute, parce que le pape avait ordonné si sévèrement l'observation des décisions du concile et que tous avaient eu assez de prudence pour ne pas amener de nouveaux conflits. La question des droits de chacun restait d'ailleurs toujours imprécise. Conrad eut le bonheur de terminer le différend, quelques années plus tard, quand il fut envoyé comme légat en Languedoc. Les statuts du chapitre général de l'an 1223 interdisent aux moines, sous les peines les plus graves, d'en appeler à un pouvoir supérieur quand le chapitre général a prononcé. Les privileges qui étaient contraires aux résolutions des chapitres étaient cassés, et l'on allait jusqu'à prononcer l'anathème contre les violateurs de cette règle. Les abbés approuvèrent de même un accord dont Conrad était l'auteur, et qui rétablissait la concorde entre les abbés de Cîteaux et de Clairvaux (2). En reconnaissance des grands services rendus a l'ordre, on décida, en I $22 \mathrm{I}$, quand il reçut l'importante mission de légat dans le Sud de la France, que dans tous les monastères, des prières seraient faites pour lui ( ${ }^{3}$ ).

\section{( $A$ continuer.)}

D. Ambroise Clement.

r. Pour les privileges ainsi obtenus, voir Pressutti, Reg. Hon. $I I I, 286,290,291,293$. - Manrique, IV, 145, 146, 185; - Potthast, op. cit., nos 5929, 5941, 5944, 5950, 5951. Pour le sens du mot mortuarium, voir du Cange, Glossariwm medie of infince latiritatis.

2. Martène et Durand, IV, pp. $1334,35,36$.

3. Ip., Ibid., p. 1330. Lorsqu'il fut élevé au cardinalat, Conrad voulut aroir tonjonrs avec lui un moine de son ordre. Il s'adressa a l'abbe de Casamari, en Italie. Sa demande fut asset mal accueiliie, l'abbé lui répondit même peu respectueusement. Pour pénitence, il dut, d'après une décision de 1219, faire six jours de coulpe, dont deux, au pain et a l'eau, et quitter au chour la stalle abbatiale pendant 40 jours. (Martene et Durand, IV, I324-25.) 